\title{
Measuring the Atomic and Electronic Structure of Black Phosphorus with STEM
}

Ryan J. Wu ${ }^{1}$, Mehmet Topsakal ${ }^{1}$, Matt C. Robbins ${ }^{2}$, Nazila Haratipour ${ }^{2}$, Jong Seok. Jeong ${ }^{1}$, Renata M. M. Wentzcovich ${ }^{1}$, Steven J. Koester ${ }^{2}$, K. Andre Mkhoyan ${ }^{1}$

${ }^{1}$ Department of Chemical Engineering and Material Science

${ }^{2}$ Department of Electrical and Computer Engineering

University of Minnesota, Minneapolis, MN, USA

Black phosphorus, a layered two-dimensional crystal with tunable electronic properties and high hole mobility, is quickly emerging as a promising candidate for future electronic and photonic devices [1]. Although theoretical studies using $a b$ initio calculations have tried to predict its atomic and electronic structure [2,3], uncertainty in its fundamental structural properties due to a lack of clear experimental evidence continues to stymie our full understanding and application of this novel material.

In this work, aberration-corrected scanning transmission electron microscopy (STEM) is used to record annular dark-field (ADF) images of few-layer black phosphorus. Directly interpretable atomicresolution ADF-STEM images captured at the [001], [101], and [100] zone axes, as shown in Figure 1, provides a three-dimensional view of this layered material which allows all three lattice parameters to be measured. The ADF-STEM images also unambiguously identified its stacking order which differs from other possible arrangements discussed in literature [3].

Furthermore, STEM monochromated electron energy-loss spectroscopy (STEM-EELS) is used to measure the conduction band density of states (DOS) of black phosphorus. The core-loss EELS measured $\mathrm{P} \mathrm{L}_{3}$-edge agrees well with density functional theory (DFT)-based calculations performed for the experimentally determined black phosphorus crystal, as shown in Figure 2a. Simultaneously collected low-loss EEL spectrum (Figure 2b) also shows energies of bulk and surface plasmons and changes with the number of layers.

Finally, the effects of oxidation on both the atomic and electronic structure of black phosphorus are analyzed to explain observed device degradation. STEM-energy dispersive X-ray (STEM-EDX) maps show that black phosphorus transforms into amorphous $\left(\mathrm{H}_{3}\right) \mathrm{PO}_{3}$ during oxidation which may ultimately be responsible for the degradation of black phosphorus-based devices exposed to atmosphere over time.

\section{References:}

[1] L. Li et al., Nat Nano 9, (2014), p. 372-377

[2] J. Qiao et al., Nat. Commun 5, (2014).4475

[3] J. Dai and X.C. Zeng, J. Phys. Chem. Lett. 5, (2014). p.1289-1293

[4] This work was supported in part by C-SPIN, one of the six centers of STARnet, a Semiconductor Research Corporation program, sponsored by MARCO and DARPA; by the NSF award DMR-0819885; and by the Defense Threat Reduction Agency award HDTRA1-14-1-0042. Computational resources were partly provided by Blue Waters sustained-petascale computing project, supported by the NSF awards OCI-180725070 and ACI-1238993 and the state of Illinois. STEM analysis was carried out in the Characterization Facility of the University of Minnesota, which receives partial support from NSF through the MRSEC program. 


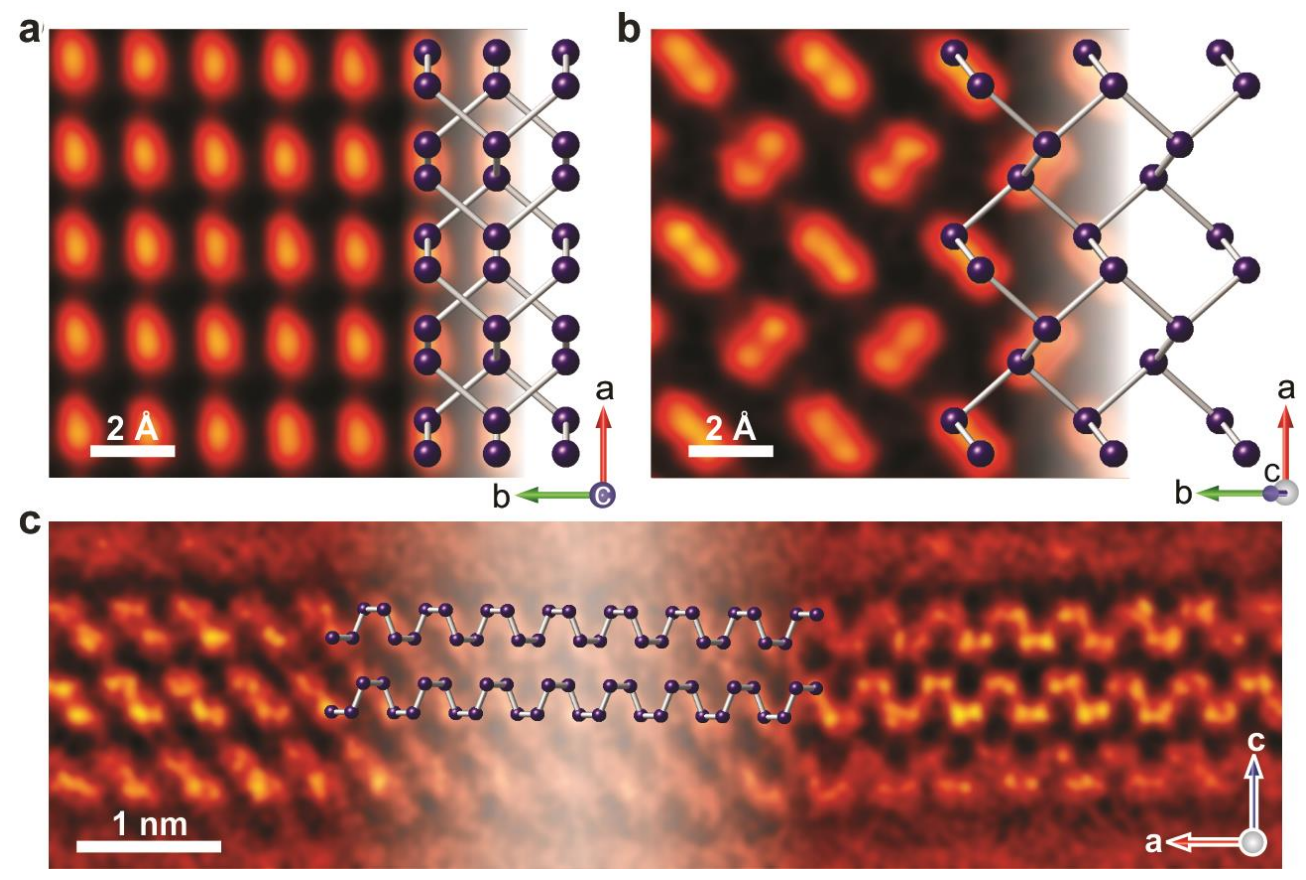

Figure 1: Atomic-resolution ADF-STEM images of black phosphorus. a, ADF-STEM image of black phosphorus viewed along the [001] crystallographic direction, or top-down view. b, ADF-STEM image viewed along the [101] direction or $17^{\circ}$ tilted off the [001] zone axis. c, ADF-STEM image captured at an edge of a black phosphorus flake showing multiple layers stacked together, or along [100] direction. Images in $\mathbf{b}, \mathbf{c}$ and $\mathbf{e}$ have overlaid ball-stick atomic models to accentuate atomic columns.
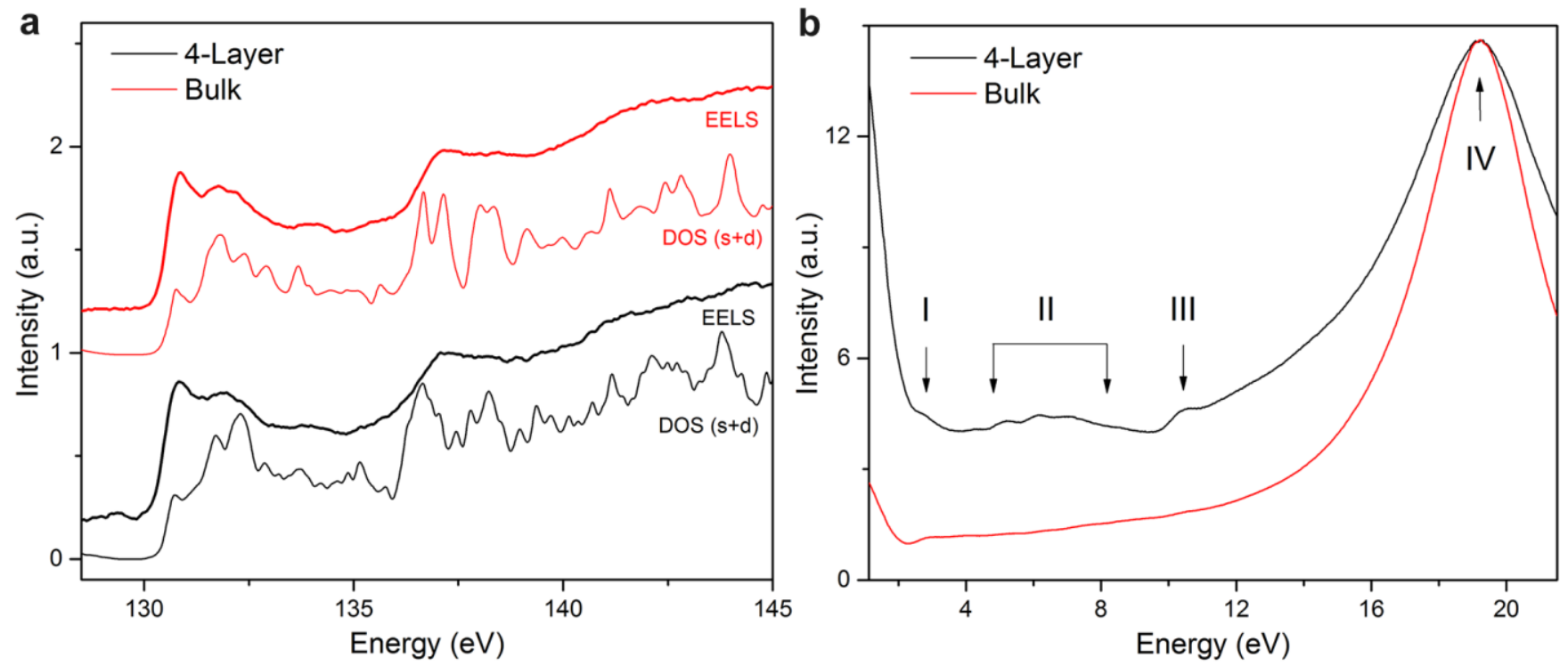

Figure 2. Electronic structure of black phosphorus. a, Monochromated EELS P $L_{3}$ edge recorded with $0.25 \mathrm{eV}$ energy resolution compared with calculated $3 s+3 d$ partial DOS. DOS data is broadened with a $0.25 \mathrm{eV}$ FWHM Gaussian function for better comparison. b, Low-loss EEL spectra from 4-layer and bulk black. Peaks I, II and III are mainly surface plasmon modes while peak IV is the bulk plasmon. 